\title{
Gradhiva
}

GRADHIV

Revue d'anthropologie et d'histoire des arts

18 | 2013

Le monde selon l'Unesco

\section{Comment le patrimoine mondial de l'Unesco devient immatériel}

Cultivating elusiveness: the intangibilisation of UNESCO World Heritage

\section{Christoph Brumann}

Traducteur : Camille Joseph

\section{OpenEdition}

Journals

Édition électronique

URL : http://journals.openedition.org/gradhiva/2698

DOI : 10.4000/gradhiva.2698

ISSN : 1760-849X

\section{Éditeur}

Musée du quai Branly Jacques Chirac

Édition imprimée

Date de publication : 1 décembre 2013

Pagination : 22-49

ISBN : 978-2-35744-072-2

ISSN : 0764-8928

\section{Référence électronique}

Christoph Brumann, «Comment le patrimoine mondial de l'Unesco devient immatériel », Gradhiva [En ligne], 18 | 2013, mis en ligne le 01 décembre 2016, consulté le 30 avril 2019. URL : http:// journals.openedition.org/gradhiva/2698; DOI : 10.4000/gradhiva.2698 



\section{Comment le patrimoine mondial de l'Unesco devient immatériel}

par Christoph Brumann

La Convention du patrimoine mondial de I'Unesco, qui constitue aujourd'hui le système de conservation du patrimoine le plus influent au niveau international, accorde une place croissante aux aspects immatériels. À côté des sites associés à des idées, des événements (tragiques) et des personnages célèbres, la nouvelle catégorie des paysages culturels permet d'inclure des sites spirituels importants pour les peuples indigènes. En outre, la réévaluation des critères d'authenticité a entraîné la réhabilitation des projets de reconstruction. De la même manière, les biens en série, qui sont de plus en plus populaires, reposent eux aussi sur des «idées » et non sur la matérialité de chaque site. Cependant, cette réorientation n'est que partielle:

l'impulsion continue de venir des États-nations, toujours aussi avides d'inscrire des sites

sur la liste du patrimoine mondial. 


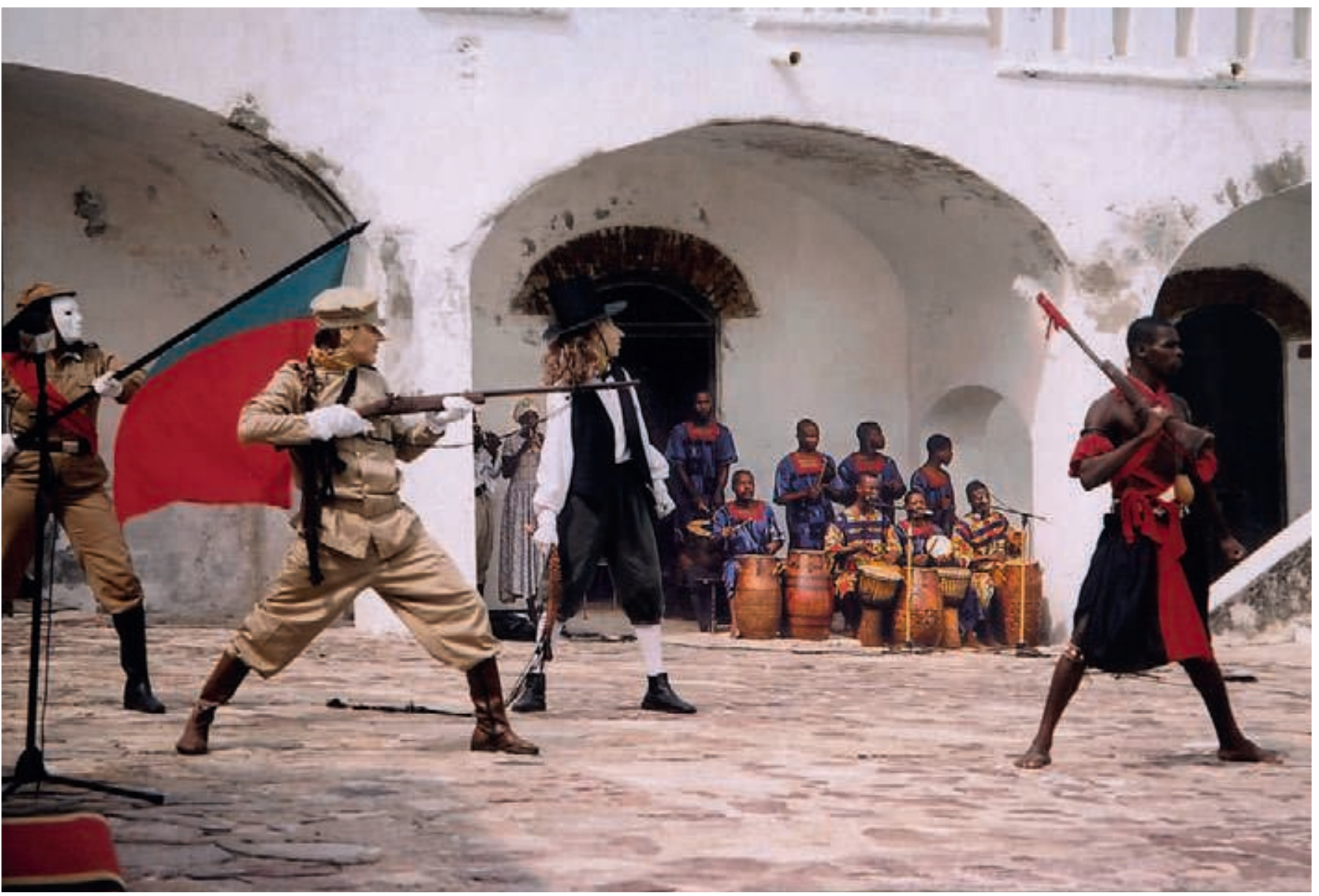

page précédente et ci-dessus

fig. 1

Le fort de Cape Coast.

Spectacle donné à l'occasion du lancement de l'année de commémoration de la lutte contre l'esclavage et de son abolition, 2004, Ghana. (C) Bernard Jacquot/Unesco.
L'Unesco est aujourd'hui un acteur décisif dans l'économie politique mondiale du patrimoine, mais il devient de plus en plus difficile d'identifier avec précision ce que recouvre ce concept pour l'Organisation. Cela n'est pas simplement dû au fait que celle-ci a récemment étendu son action au patrimoine culturel immatériel, dont l'existence a été reconnue une première fois par la Proclamation des chefs-d'œuvre du patrimoine oral et immatériel de l'humanité puis par l'adoption, en 2003, d'une Convention pour la sauvegarde du patrimoine culturel immatériel (sous la forme d'un traité international ayant force exécutoire). La Convention sur la protection du patrimoine mondial, culturel et naturel, qui a fêté son quarantième anniversaire en 2012 et a été ratifiée par 189 États (soit presque la totalité d'entre eux), met elle aussi de plus en plus l'accent sur des aspects de la production culturelle qui ne sont pas immédiatement perceptibles et mesurables. Cependant, cette reconnaissance n'est que partielle: les déclarations officielles insistent bien souvent sur la distinction entre les deux conventions, le patrimoine mondial restant ainsi solidement ancré dans le domaine du tangible. Cet article cherche à montrer comment les réorientations récentes des politiques de l'Unesco renforcent la dimension immatérielle du patrimoine mondial et à déterminer les causes d'une telle évolution.

Cette réflexion tombe à point nommé puisque, si la nouvelle Convention et sa liste de rites, compétences et performances font l'objet 
d'un intérêt anthropologique certain, le patrimoine mondial, de loin le programme le plus populaire de l'Unesco, reste plus important, non seulement pour les pays du Sud, mais également pour les pays occidentaux, héritiers d'une noble histoire de la conservation institutionnalisée. En effet, l'inscription au patrimoine mondial contribue souvent à développer le tourisme, la fierté nationale et le développement local; elle peut favoriser des projets de conservation et mobiliser des soutiens politiques et financiers considérables. Identifier les prémisses cachées de ces programmes ne permet pas seulement de mieux comprendre ce qui continue d'être le modèle de politique patrimoniale le plus puissant, mais également de saisir les relations complexes qui lient les deux conventions de l'Unesco et influencent de manière décisive leurs trajectoires respectives ${ }^{\mathbf{1}}$.

\section{Le critère (vı)}

Depuis l'adoption de la Convention en 1972 et l'inscription des premiers sites sur la liste du patrimoine mondial en 1978, I'Unesco s'est focalisée sur des espaces. Les 962 biens $^{2}$ du patrimoine mondial correspondent (ne serait-ce que dans l'idéal) à des zones bien délimitées de territoire ou de mer ou à des combinaisons de plusieurs zones - allant parfois jusqu'à une centaine. L'inscription au patrimoine mondial prend généralement en compte la faune, la flore et les éléments fixes présents dans ces espaces, mais elle laisse de côté les idées, les pratiques et les artefacts mobiles. Dans une majorité de cas, l'accent est mis sur les bâtiments et leurs vestiges. Ainsi, tout ce qui est censé avoir une «valeur universelle exceptionnelle», ou VUE, est normalement visible par quiconque visite le site en question (le critère de la VUE, valable pour l'humanité tout entière et pas seulement pour certaines factions nationalistes, religieuses ou autres, représente la condition préalable à l'inscription sur la liste du patrimoine mondial).

Lors de mes discussions avec des acteurs du patrimoine mondial, j'ai entendu dire à plusieurs reprises que certains lieux ne peuvent qu'émouvoir ceux qui les contemplent: ce sont des sites sensationnels tels que le Grand Canyon ou les pyramides de Gizeh. On est cependant en droit de se demander s'ils sont véritablement en mesure d'impressionner n'importe qui sur cette planète, même s'il faut bien admettre que de nombreux sites figurant sur la liste du patrimoine mondial sont visuellement grandioses et attirent des millions de visiteurs. Leur succès repose bien évidemment sur certaines normes culturelles, mais la Convention sur le patrimoine mondial, en tant que vecteur de la mondialisation, encourage elle-même la diffusion de ces standards et, par suite, leur uniformisation. À côté de ces sites spectaculaires, il existe toutefois des biens plus "discrets» dont l'intérêt tient au rapport qu'ils entretiennent avec des événements, des idées ou des personnages importants; cela ne transparaît pas dans leur aspect extérieur et doit faire l'objet d'une explication. Dès ses origines, le Comité du patrimoine mondial - l'instance décisionnelle principale - a pris des dispositions concernant ces lieux grâce au critère (vi), l'un des six critères culturels de la VUE figurant dans le paragraphe 77 des Orientations ${ }^{3}$, le «code juridique» qui a peu à peu été élaboré pour la mise en œuvre de la Convention. Dans la version actuelle, on peut lire:
1. Mes recherches

sur l'univers du patrimoine mondial combinent des analyses de documents des observations participantes à des réunions et événements officiels, ainsi que des entretiens avec certains acteurs décisifs des organismes en question. J'ai suivi toutes les sessions du Comité du patrimoine mondial et de l'Assemblée générale de 2009 à 2012, ainsi que plusieurs autres rassemblements. J'ai parfois utilisé ces occasions pour conduire des entretiens, mais la plupart ont été réalisés lors de séjours de recherche organisés à part. Je suis très reconnaissant à la Fondation allemande à la recherche (DFG) pour son financement en 2008-2010, et au Max Planck Institute for Social Anthropology à Halle pour son soutien à mon groupe de recherche actuel, «The Global Political Economy of Cultural Heritage".

Pour d'autres incursions dans les procédures décisionnelles relatives au patrimoine mondial, je renvoie à Schmitt (2009, 2011), Rudolff (2010) et Turtinen $(2000,2006)$; pour une étude sur la genèse et les débuts de l'histoire du patrimoine mondial, voir Titchen (1995).
2. La liste de tous les biens et informations détaillées les concernant est accessible sur le site http://whc.unesco. org/fr/list.

3. Pour les versions actuelle et antérieures, voir http://whc. unesco.org/fr/orientations. 


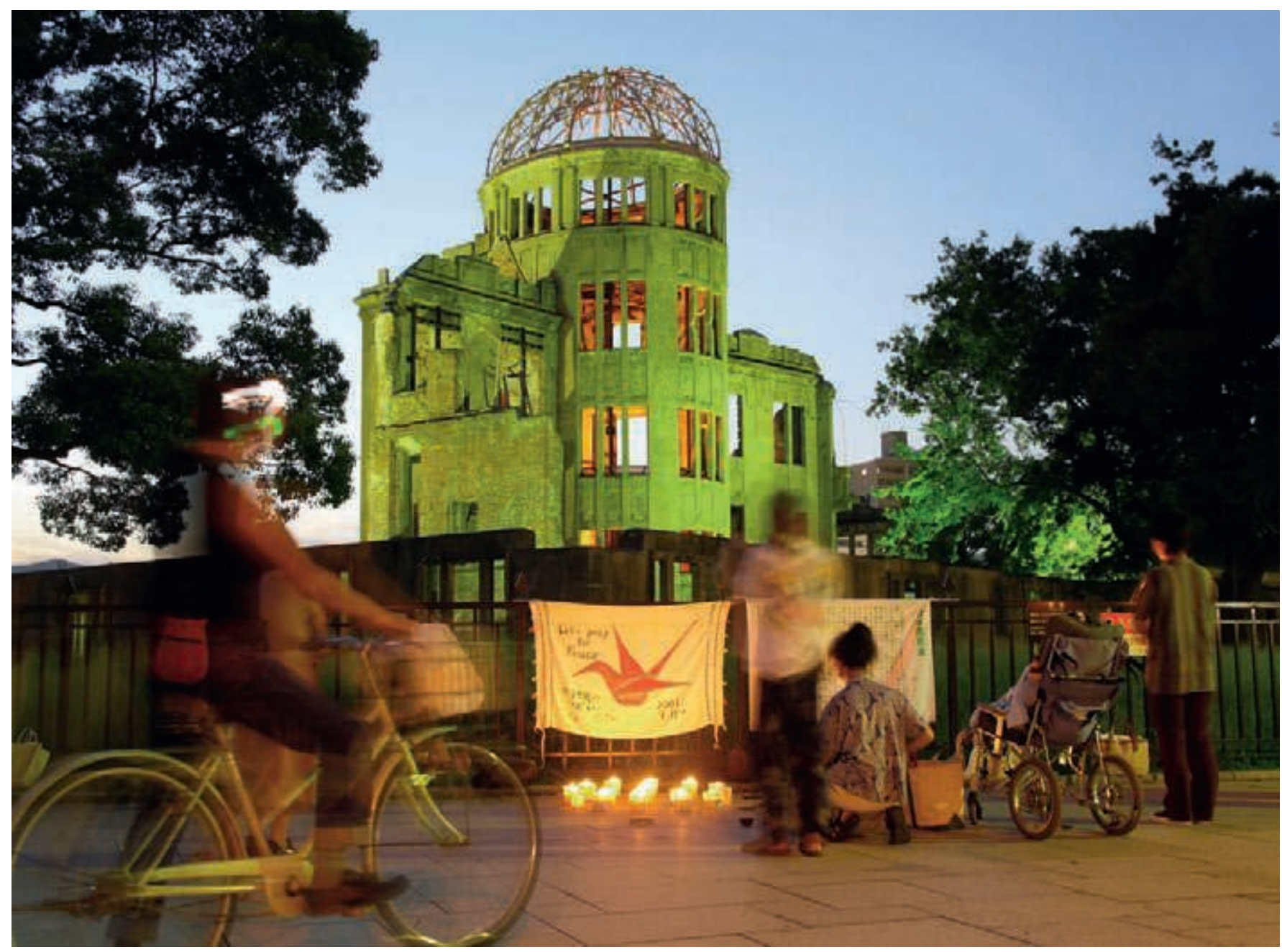

fig. 2

Mémorial de la Paix

d'Hiroshima, dôme de

Genbaku, s.d. OURPLACE

Collection. () Photo de Geoff

Steven. 
$\S 77$ [L]es biens proposés doivent [...] (vı) être directement ou matériellement associés à des événements ou des traditions vivantes, des idées, des croyances ou des œuvres artistiques et littéraires ayant une signification universelle exceptionnelle (le Comité considère que ce critère doit de préférence être utilisé conjointement avec d'autres critères ${ }^{4}$ ).

La plupart des biens du patrimoine mondial sont inscrits sous deux ou plusieurs critères. Cependant, dès la création du critère $(\mathrm{vl})^{\mathbf{5}}$, le Comité a répertorié certains sites sous ce seul critère, dont pas moins de cinq durant les deux premières années. Trois d'entre eux, classés dans le «patrimoine sombre", sont liés à la traite négrière (l'île de Gorée au Sénégal ainsi que les forts et châteaux coloniaux du Ghana) et à l'Holocauste (Auschwitz-Birkenau en Pologne ${ }^{6}$ ). Auschwitz fut qualifié de cas exceptionnel ayant valeur d'exemplarité pour d'autres sites semblables ${ }^{7}$ afin d'éviter que la liste, qui visait aussi à célébrer les réalisations positives de l'humanité, ne soit remplie de camps de concentration. Les difficultés commencèrent dès 1979 avec la proposition américaine d'inscrire sur la liste le laboratoire et domicile de Thomas Edison. La proposition fut rejetée l'année suivante: on craignit en effet de créer un précédent pour des biens dont le mérite serait d'être eux aussi associés à des personnages célèbres, et d'encourager ainsi certains nationalismes et autres particularismes. Par la même occasion, le Comité déclara que le critère (vI) ne devait être invoqué que de manière exceptionnelle ou bien combiné à d'autres critères. II ne fut utilisé seul que jusqu'en 1996, date de l'inscription du Mémorial de la Paix d'Hiroshima ${ }^{8}$. Cependant, dans de nombreux cas $^{9}$, il fut invoqué conjointement à d'autres critères. En 1997, I'utilisation combinée du critère (vi) devint même obligatoire. Puis, en 2001, après un long débat au cours duquel les États africains, en particulier, s'alarmèrent du fait que, sans la possibilité d'utiliser le critère (vi) seul, le patrimoine architectural européen continuerait à être surreprésenté sur la liste, le Comité adopta une formule plus flexible: il était désormais fait mention d'une simple préférence pour une utilisation combinée du critère ${ }^{\mathbf{1 0}}$.

Si le critère (VI) reste ainsi le cousin pauvre des autres critères, les propositions qui s'en réclament sont bien souvent celles qui mènent aux discussions les plus complexes et les plus passionnées au sein du Comité. Cela est particulièrement vrai pour un certain nombre de sites qui, à l'instar d'Auschwitz et des forts de la traite négrière, ne commémorent pas uniquement la mémoire de l'oppression et des atrocités commises par le passé dans certains «lieux de la douleur et de la honte» (Logan et Reeves 2008), mais célèbrent également, de manière implicite, les progrès de l'humanité jusqu'à un stade de développement où celle-ci prend conscience de ses erreurs et tire des leçons de leurs témoignages matériels. Avec l'inscription controversée du site d'Hiroshima - qui fit l'objet de fortes objections de la part des États-Unis et de la Chine $\mathbf{1 1}^{\mathbf{1 1}}$ et celle, moins polémique, du site d'essais nucléaires de l'atoll de Bikini (2010), on aborda la question de la guerre nucléaire. L'éradication des haines raciales, ethniques et religieuses fut quant à elle commémorée avec l'inscription au patrimoine mondial de la prison de Robben Island où Nelson Mandela fut enfermé (1999), de la vieille ville de Mostar (2005) et de son célèbre pont, qui, après sa destruction délibérée pendant la guerre des Balkans, venait d'être reconstruit
4. Selon les autres critères culturels actuellement en vigueur, les biens doivent: "[...] (ı) représenter un chefd'œuvre du génie créateur humain; (II) témoigner d'un échange d'influences considérable pendant une période donnée ou dans une aire culturelle déterminée, sur le développement de l'architecture ou de la technologie, des arts monumentaux, de la planification des villes ou de la création de paysages; (III) apporter un témoignage unique ou du moins exceptionnel sur une tradition culturelle ou une civilisation vivante ou disparue; (Iv) offrir un exemple éminent d'un type de construction ou d'ensemble architectural ou technologique ou de paysage illustrant une période ou des périodes significative(s) de l'histoire humaine; (v) être un exemple éminent d'établissement humain traditionnel, de l'utilisation traditionnelle du territoire ou de la mer, qui soit représentatif d'une culture (ou de cultures), ou de l'interaction humaine avec l'environnement, spécialement quand celui-ci est devenu vulnérable sous l'impact d'une mutati on irréversible [...]. »
5. Pour une histoire détaillée de l'usage du critère (vI), voir WHC-01/CONF.208/ INF.13. Désormais, je ferai référence aux documents de l'Unesco avec leur code d'identification permanent. Ces textes sont tous disponibles en ligne et faciles à retrouver grâce à une recherche par code, quand bien même les URL correspondants auraient changé entre-temps.

6. WHC-01/CONF.208/ INF.13, p. 2-3.

7. CC-79/CONF.003/13, p. 11.

8. CC-79/CONF.005/06, p. 4 ; WHC-01/CONF.208/ INF.13, p. 2-3.

9. Ibid., p. 22.

10. Ibid., p. 4-6.

11. WHC-96/CONF.201/21, p. 69. 


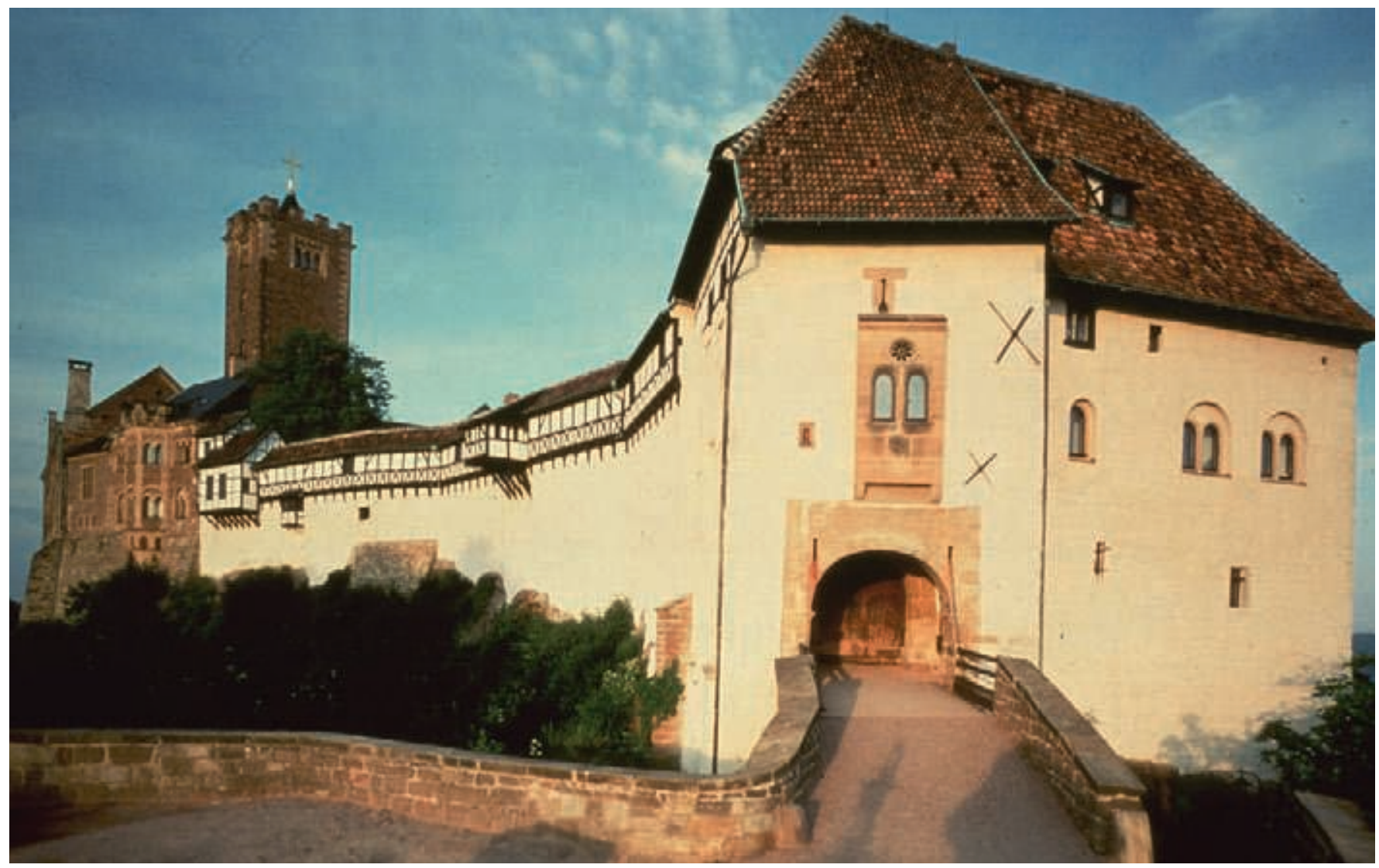

fig. 3

La Wartburg, poterne et pont-levis, classés en 1999. (c) Unesco.
12.SC/83/CONF.009/8, p. 11; WHC-01/CONF.208/23; WHC-01/CONF.208/24, p. 4-7.

13. WHC- $06 / 30 . \mathrm{COM} /$ INF.8B.1, p. 24-26.

14. WHC-08/32.COM/ INF.8B1, p. 2 sous le patronage de l'Unesco, et de la vallée de Bâmiyân (2003), deux ans après que les talibans eurent dynamité les deux grands Bouddhas. Dans ce dernier cas, il s'agissait d'une forme de réparation suite au refus de l'Unesco d'inscrire le site en $1983^{12}$. Dans chacune de ces inscriptions, des éléments immatériels - le symbolisme politique des sites - jouèrent un rôle décisif. Dans le débat très passionné qui entoura l'inscription de Robben Island, le Comité finit par faire référence à un autre critère quelque peu improvisé; Bâmiyân fut répertorié en tant que paysage culturel (voir ci-dessous), ce qui permettait d'étendre l'intérêt du site au-delà des niches vides des Bouddhas et de justifier ainsi le recours à d'autres critères. Cependant, dans le cas de Mostar, l'inscription reposa sur le seul critère (vI).

Dans la liste des biens «politiques» évoqués plus haut, il existe une sous-catégorie associée aux migrations forcées. Aux sites esclavagistes répertoriés au cours des premières années en furent ajoutés deux situés sur l'île Maurice: Aapravasi Ghat (2006), le lieu d'arrivée d'un demi-million de travailleurs sous contrat venus d'Inde pour travailler dans les plantations sucrières, et Le Morne (2008), une montagne accidentée autrefois utilisée comme refuge par les Marrons. Lors de sa session de 2010, le Comité valida l'inscription des sites de bagnes australiens. Dans tous les cas mentionnés ici, le critère ( $\mathrm{vl}$ ) fut associé à d'autres, mais tant pour Aapravasi Ghat - où le site est dans un état de grand délabrement ${ }^{13}$ - que pour Le Morne - où il reste peu de traces matérielles de la présence des Marrons ${ }^{\mathbf{1 4}}$-, les aspects intangibles jouèrent manifestement un rôle décisif. 


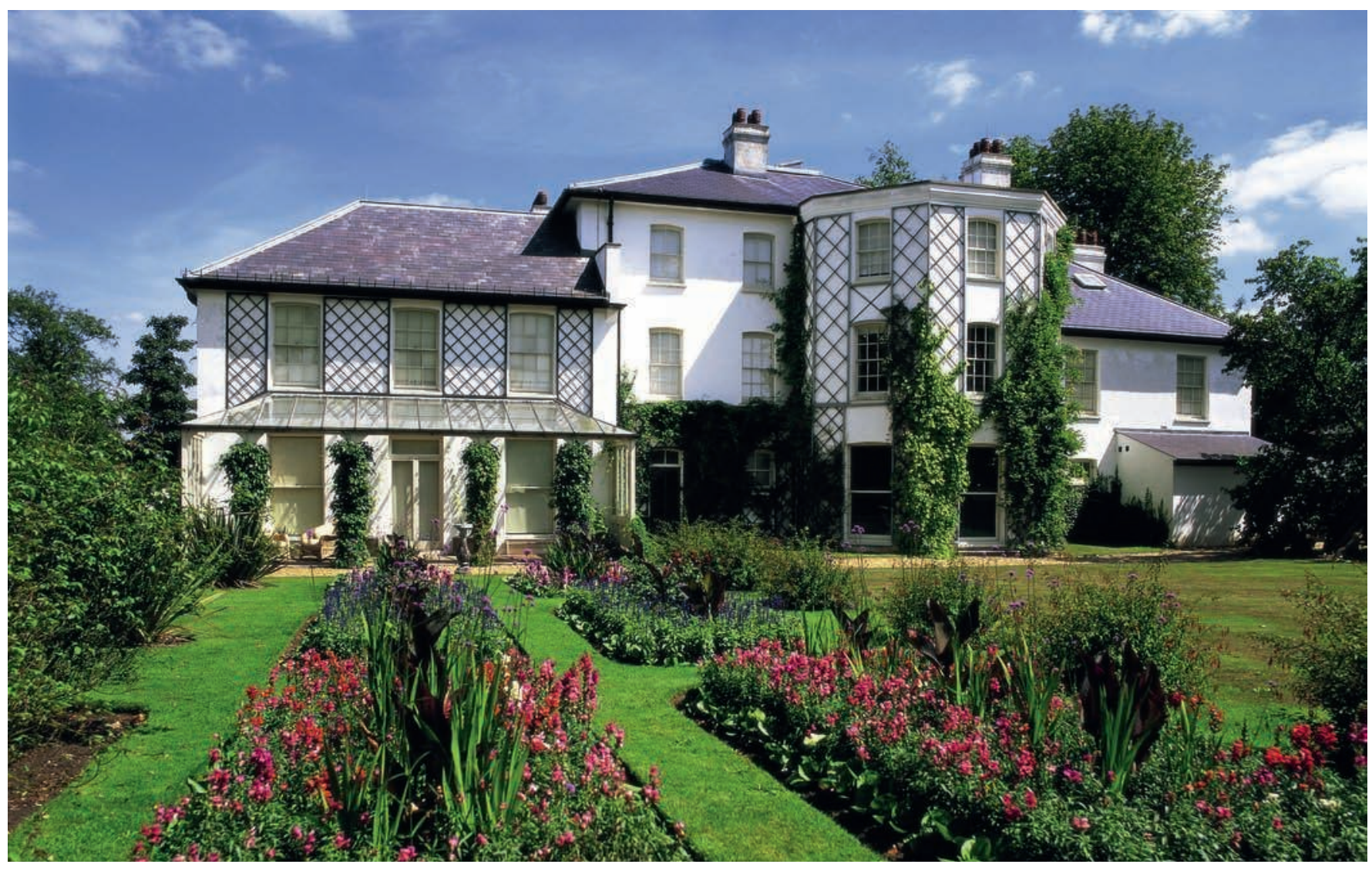

Si l'on regarde maintenant au-delà du patrimoine "sombre", la même chose se produisit pour les lieux saints bahá'i à Haïfa, en Israël. En 2007, après l'enregistrement de la proposition d'inscription, l'lcomos - le Conseil international des monuments et des sites, organisme de conseil pour les biens culturels - finit par se déclarer incompétent pour évaluer la candidature. Les bâtiments proposés à l'inscription étaient plutôt récents; seul pouvait donc être évalué le dogme bahá'i dont l'Icomos, en tant qu'instance experte du patrimoine, ne se sentit pas capable de juger de la pertinence ${ }^{\mathbf{1 5}}$. Lors d'une seconde proposition, l'accent fut mis sur un plus petit nombre de sites dédiés à une tradition mondiale de pèlerinage, ce qui permit de préparer le terrain à l'utilisation d'un autre critère et, finalement, à leur inscription en 2008.

Les grands hommes ne furent pas en reste. En 2007, I'Icomos s'opposa de manière catégorique à l'inscription de Down House, la maison du Kent où Charles Darwin fit un certain nombre de découvertes capitales et écrivit Sur l'origine des espèces. Les raisons étaient que, le bâtiment ayant été en grande partie restauré, il ne répondait pas aux critères d'authenticité nécessaires, et que le lien avec Darwin lui-même n'était pas jugé suffisamment fort ${ }^{16}$. Le Comité décida de renvoyer la proposition, ce qui permit d'en présenter une autre, profondément révisée, en 2010. Cette fois-ci, elle concernait le "paysage laboratoire» qui comprenait l'espace environnant la maison, dont la diversité géologique et biologique avait attiré Darwin. L'Icomos s'opposa une nouvelle fois au projet, arguant que cet espace n'était qu'une campagne anglaise ordinaire et ne présentait aucune trace
15. WHC-07/31.COM/ INF.8B1, p. 181.

16. Ibid., p. 228 fig. 4

Down House, la demeure de Charles Darwin, Luxted Road Downe Kent England, s.d. (C) David Ball/Alamy. 
17. Orientations, 2008 , annexe III, § 10.

18. WHC-01/CONF.208/ INF.13, p. 3.

19. Orientations, 2008 , annexe III, § 10.

20. WHC-08/32.COM/ INF.8B1, p. 93. matérielle des activités du savant. Dans ce raisonnement, l'accent est avant tout mis sur les aspects tangibles qui, d'une certaine manière, doivent permettre d'établir un lien physique avec un personnage ou un événement. Ce point de vue fit l'objet d'un débat passionné au sein du Comité, qui finit par se prononcer pour un nouveau renvoi, laissant ainsi la possibilité à Down House d'être proposée une autre fois pour inscription.

Si l'on suit le raisonnement de l'Icomos dans le cas de la maison de Darwin, le site Edison aurait dû figurer sur la liste puisque, à la différence de Down House, le laboratoire du savant américain n'a subi aucune altération depuis sa fondation. Dans plusieurs autres cas, le lien avec des personnages célèbres joua manifestement un rôle déterminant ( $y$ compris pour Robben Island, mentionnée ci-dessus). En Allemagne, le château de la Wartburg, inscrit en 1999, fut reconnu comme un des «monuments remarquables de la période féodale en Europe centrale». Pourtant, la structure du bâtiment, entièrement rénovée, date du xIx ${ }^{e}$ siècle. Le critère (vi) fut pris en compte, le lieu étant associé à un personnage célèbre et à une religion: c'est là, dans des parties du château aujourd'hui détruites, que Martin Luther, alors en exil, traduisit la Bible. Cet épisode fut sans nul doute fondamental dans l'histoire du protestantisme, mais ce qui se déroula à Down House ne fut pas moins décisif pour l'évolution des sciences naturelles; à moins d'accorder un poids prépondérant à la dimension religieuse, on serait bien en peine de relever ici une quelconque cohérence.

\section{Paysages culturels}

Les aspects immatériels se sont également fait une place au sein du patrimoine mondial à travers la création, en 1992, d'une nouvelle catégorie: les paysages culturels (Fowler 2003). Ici, c'est l'interaction entre les hommes et leur environnement qui est centrale. Aux paysages artificiels comme les parcs et aux paysages évolutifs tels que les régions vinicoles vient s'ajouter la sous-catégorie des «paysages culturels associatifs», caractérisés par «la force d'association des phénomènes religieux, artistiques ou culturels de l'élément naturel ${ }^{\mathbf{1 7}}$ ». Les deux premiers paysages culturels inscrits sur la liste du patrimoine mondial, les parcs nationaux de Tongariro en NouvelleZélande (1993) et d'Uluru-Kata Tjuta (Ayers Rock) en Australie (1994), relèvent de cette dernière catégorie. En raison de l'importance spirituelle de ces sites pour les populations indigènes locales, le critère (vi) fut ajouté au critère naturel ayant initialement servi à leur inscription quelques années plus tôt ${ }^{18}$.

Dans le cas des paysages culturels associatifs, les «traces culturelles matérielles [...] peuvent être insignifiantes ou même inexistantes ${ }^{19}$ "; comme nous l'avons dit plus haut, les Marrons laissèrent peu de traces physiques de leur passage sur la montagne du Morne, elle aussi répertoriée comme paysage culturel. Le domaine du chef Roi Mata, récemment inscrit sur la liste (2008), présente des traces d'activité humaine - une peinture rupestre qui représenterait le chef, un arbre qu'il aurait planté et un squelette enterré dans une position exceptionnelle qui serait sa dépouille mortelle -, sans toutefois que le lien entre ces lieux et le dernier détenteur du titre de chef de Vanuatu ait été établi avec certitude ${ }^{20}$. La VUE du site repose en réalité sur des traditions orales évoquant le Roi Mata dont l'importance 
est aujourd'hui capitale dans le Vanuatu central ${ }^{21}$; de la même manière, la VUE du site du Morne s'appuie sur les traditions orales relatant l'histoire des Marrons et le rôle remarquable joué par la montagne dans leur émancipation 22. Dans le cas du Val d'Orcia, en Toscane (2004), c'est l'influence de ce paysage culturel sur l'école siennoise de peinture de la Renaissance qui fut mise en avant, les représentations ayant là aussi tendance à primer sur le site lui-même. Bien que les œuvres d'art en question soient matérielles, elles ne font pas partie du paysage répertorié, qui présente aussi peu de marques physiques de leur existence que le paysage entourant Down House, lieu des observations de Darwin.

Les caractéristiques des paysages culturels associatifs sont si éphémères qu'on peut légitimement s'attendre à ce que les deux conventions de I'Unesco finissent un jour par converger. Les onze forêts sacrées de kayas des Mijikenda au Kenya, inscrites en 2008, ont ouvert la voie puisque le Comité du patrimoine mondial a dans ce cas évoqué le rôle capital joué par ces lieux dans les croyances et le culte des ancêtres mijikenda. Un an plus tard, les «traditions et pratiques associées aux kayas dans les forêts sacrées des Mijikenda» ont été inscrites sur la «liste du patrimoine culturel immatériel nécessitant une sauvegarde urgente» de la Convention de $2003^{23}$; c'était la première fois qu'un site figurait sur deux listes de l'Unesco à la fois. Notons que, étant donné la rivalité existant entre les deux conventions (voir plus loin), le statut de patrimoine mondial n'est pas mentionné dans le texte de la décision. Rien n'indique en outre que l'Unesco ait coordonné les deux inscriptions.

\section{Reconstructions}

Dans tous les cas évoqués jusqu'à présent, la dimension immatérielle apparaît clairement à travers la référence au critère (vi). Cependant, il me semble qu'il existe d'autres biens du patrimoine mondial où cette dimension, qui n'est pas reconnue comme telle, est pourtant manifestement présente. Parmi eux figurent les sites rénovés. Dès l'origine, il était précisé que les biens du patrimoine mondial devaient «faire la preuve de leur authenticité en termes de plan, de matériaux, d'exécution et de localisation ${ }^{\mathbf{2 4}}$ ». Cette recommandation se fondait sur la charte de Venise de $1964^{25}$, texte fondateur de la conservation architecturale moderne qui n'autorise la reconstruction que dans des circonstances exceptionnelles et quand celle-ci peut être facilement distinguée des éléments d'origine ${ }^{26}$. L'intérêt, aujourd'hui si grand, porté aux matériaux d'origine et à leur patine n'est pourtant qu'une évolution relativement récente dans I'histoire de la conservation en Occident, apparue longtemps après que John Ruskin et William Morris eurent commencé à diffuser l'idée selon laquelle «la conservation des monuments du passé n'est pas une simple question de convenance ou de sentiment. Nous n'avons pas le droit d'y toucher. Ils ne nous appartiennent pas. Ils appartiennent en partie à ceux qui les ont construits, en partie à toutes les générations d'hommes qui viendront après nous. " (Ruskin 1987 [1849]: 206) Avant eux, Eugène Viollet-le-Duc, l'un des pères fondateurs de la conservation, estimait que la vision artistique d'origine avait plus d'importance que l'exécution matérielle concrète, qui pouvait toujours être en deçà de l'idéal de départ. Ainsi, «restaurer un édifice, ce n'est pas l'entretenir, le réparer ou le refaire, c'est le rétablir dans un état complet qui peut n'avoir
21. WHC-08/32.COM/ INF.8B1, p. 96.

22. WHC-01/CONF.208/ INF.13, p. 4.

23. $\mathrm{TTH} / 09 / 4 . \mathrm{COM} /$ CONF.209/Decisions, p. 96-97.

24. Operational Guidelines, octobre 1977, § 9 [texte seulement disponible en anglais].

25. http://www.icomos.org/ charters/venice_f.pdf

26. "Article 9. La restauration [...] se fonde sur le respect de la substance ancienne et de documents authentiques. [...] Article 11. Les apports valables de toutes les époques à l'édification d'un monument doivent être respectés, l'unité de style n'étant pas un but à atteindre au cours d'une restauration. [...] Article 12. Les éléments destinés à remplacer les parties manquantes doivent s'intégrer harmonieusement à l'ensemble, tout en se distinguant des parties originales, afin que la restauration ne falsifie pas le document d'art et d'histoire. [...]" 


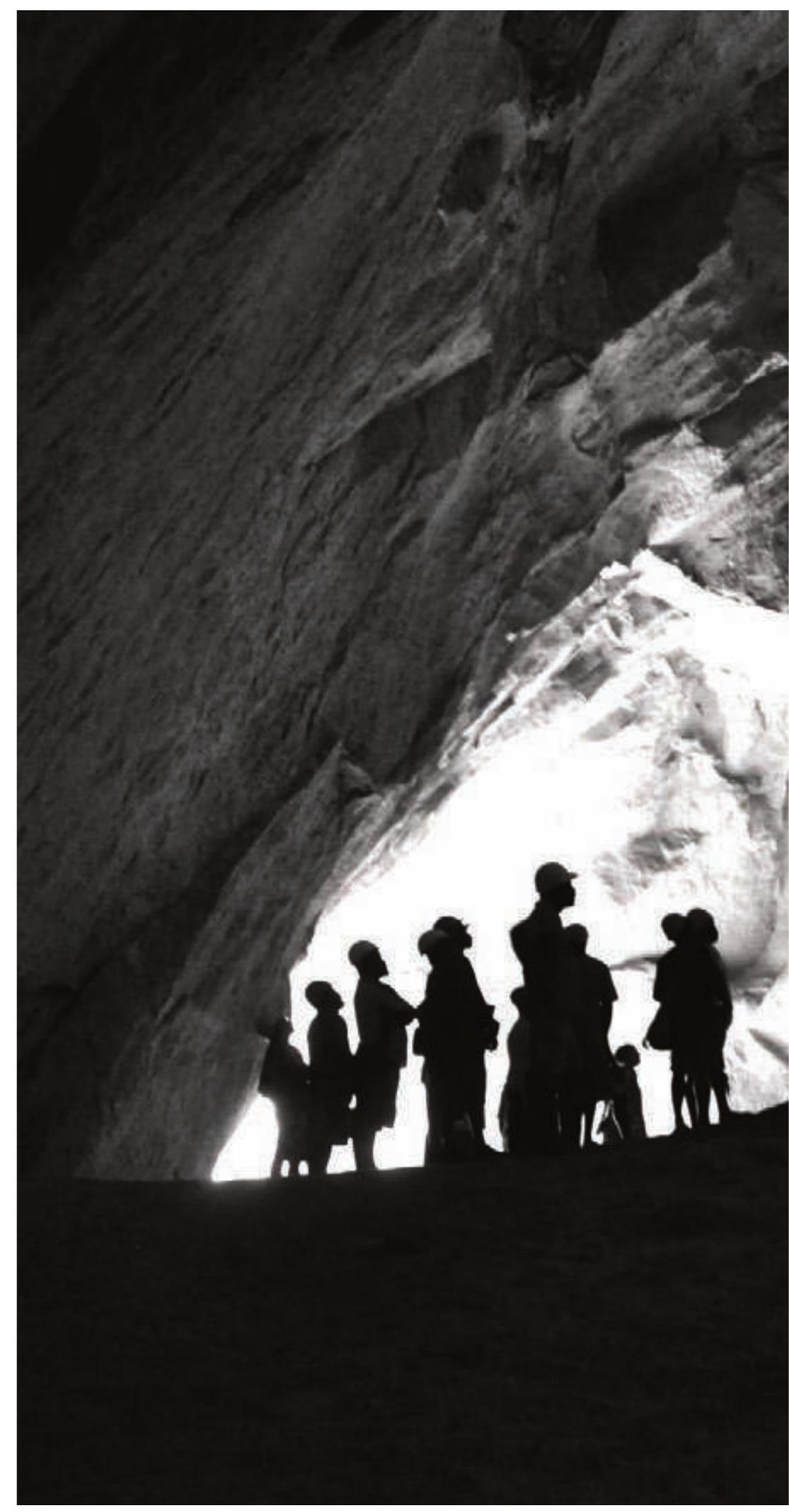




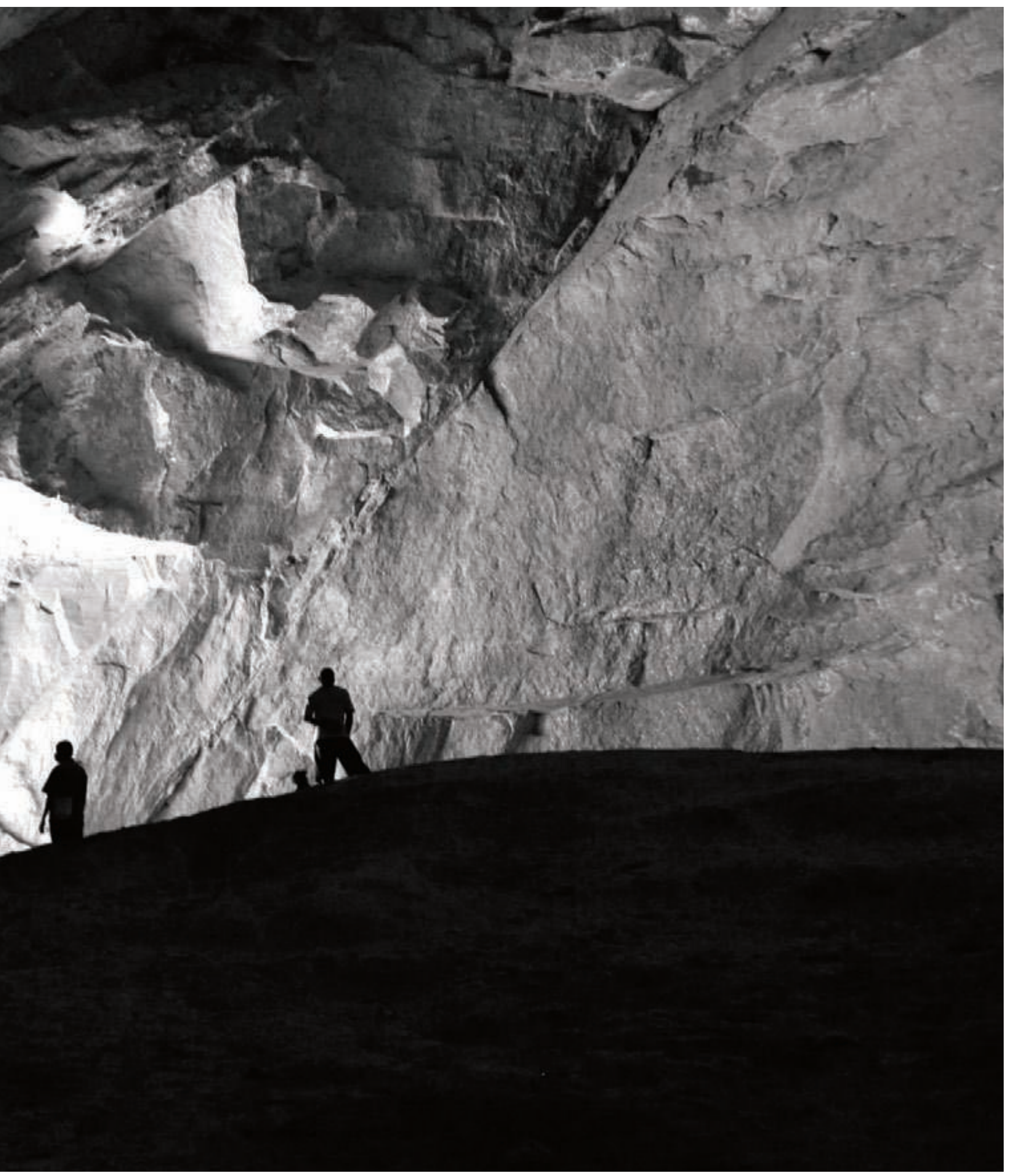


27. http://whc.unesco.org/ archive/advisory_body_ evaluation/660.pdf, p. 3-4

28. http://www.international. icomos.org/naradoc_eng.htm

29. WHC-95/CONF.203/16, p. 25.

30. WHC-94/CONF.003/16, p. $40, \mathrm{WHC}-06 / 30 . \mathrm{COM} /$ INF.19, p. 131.

31. WHC-08/32.COM/24, p. $138-139$

32. WHC-06/30.COM/19, p. 101.

33. WHC-95/CONF.203/16 p. 22-23, WHC-96/ CONF.201/21, p. 23-24, WHC-2000/CONF.202/17, p. 14.

34. http://whc.unesco.org/ archive/advisory_body_ evaluation/708.pdf, p. 16.

35. WHC-99/CONF.204/ INF.7, p. 68.

36. WHC-2000/CONF.204/ INF.6, p. 150-151.

37. WHC-2000/ CONF.204/21, p. 41.

38. WHC-02/CONF.201/ INF.2, p. 44-45.

39. WHC-04/28.COM/26, p. 217. jamais existé à un moment donné» (Viollet-le-Duc 1875: 14). Ce raisonnement, où les idées priment sur les réalisations matérielles, doit dès lors être compris comme une autre façon de privilégier l'intangible. Après avoir été mise à l'écart par une certaine orthodoxie en matière de restauration à la fin du $x^{e}$ siècle, cette conception est actuellement en train de regagner du terrain dans le champ du patrimoine mondial.

Cela s'explique en partie par le débat sur l'authenticité déclenché en 1993 par la proposition japonaise d'inscrire le site de Hôryû-ji, près de Nara; il s'agit d'un ensemble de temples parmi lesquels se trouveraient les plus anciens édifices de bois du monde, datant des vil et vill siècles. Ces bâtiments connurent d'importantes campagnes de restauration aux $\mathrm{XIII}^{\mathrm{e}}$, $\mathrm{XVII}^{\mathrm{e}}$ et $\mathrm{xx}^{\mathrm{e}}$ siècles, au cours desquelles ils furent entièrement ou partiellement démontés, et une grande partie des matériaux abîmés remplacés ${ }^{27}$. Certains experts européens remirent en question leur authenticité mais n'eurent pas gain de cause; en 1994, des spécialistes se réunirent pour rédiger le "document de Nara sur l'authenticité ${ }^{28}$ ", qui permet désormais de fonder l'authenticité d'un site sur une série de critères supplémentaires. En 2005, ces recommandations furent intégrées aux Orientations (§ 81 à § 83).

Il est bien évident que bon nombre des nouvelles conditions permettant de définir l'authenticité sont en grande partie intangibles puisqu'elles n'apparaissent pas dans la structure matérielle: «[T]raditions, techniques et systèmes de gestion; situation et cadre; langue et autres formes de patrimoine immatériel; esprit et impression [...].» (§ 82) Dès leur publication, ces nouveaux critères ont servi à justifier l'inscription des paysages culturels associatifs. Ils ont également permis de remettre en question l'orthodoxie de la charte de Venise. Celle-ci n'a toutefois pas complètement disparu: les Orientations insistent toujours sur le fait que «la reconstruction [...] n'est justifiable que dans des circonstances exceptionnelles. La reconstruction n'est acceptable que si elle s'appuie sur une documentation complète et détaillée et n'est aucunement conjecturale.» (§ 86) Par conséquent, le Comité continue de déplorer les reconstructions non conformes et les rénovations excessives de sites déjà inscrits sur la liste tels que les centres historiques du Caire ${ }^{29}$, d'Istanbul ${ }^{30}$ et de Quito ${ }^{31}$, la Cité interdite de Pékin ${ }^{32}$ et le fort de Bahla en Oman ${ }^{33}$. Si la maison de Darwin, taxée d'inauthenticité, fut rejetée en dépit du fait qu'elle fut méticuleusement reconstruite à l'identique, de plus en plus de sites reconstruits ont aujourd'hui le privilège de figurer sur la liste du patrimoine mondial. Parfois, comme ce fut le cas avec Mostar (voir plus haut), ou encore, il y a plus d'une vingtaine d'années, avec le centre historique reconstruit de Varsovie (1980), la dimension politique l'emporta sur toutes les objections possibles. Dans d'autres cas, comme avec les églises historiques de Mtskheta, l'ancienne capitale de la Géorgie ${ }^{34}$ (1994), le château de la Wartburg $^{35}$ (1999), les fortifications de Bellinzone ${ }^{36}$ (2000), le site archéologique de Tiwanaku en Bolivie ${ }^{37}$ (2000), la vallée du Haut-Rhin moyen et ses nombreux châteaux médiévaux rénovés au xix siècle $^{\mathbf{3 8}}$ (2002), et la forteresse de Kuressaare en Estonie ${ }^{39}$ (2004), l'absence d'authenticité, selon la définition de la charte de Venise, a été largement minimisée: on a jugé qu'elle était "typique de l'époque", "motivée par des facteurs religieux", "ne datant pas d'une époque récente", ou bien encore qu'elle passait à l'arrière-plan 


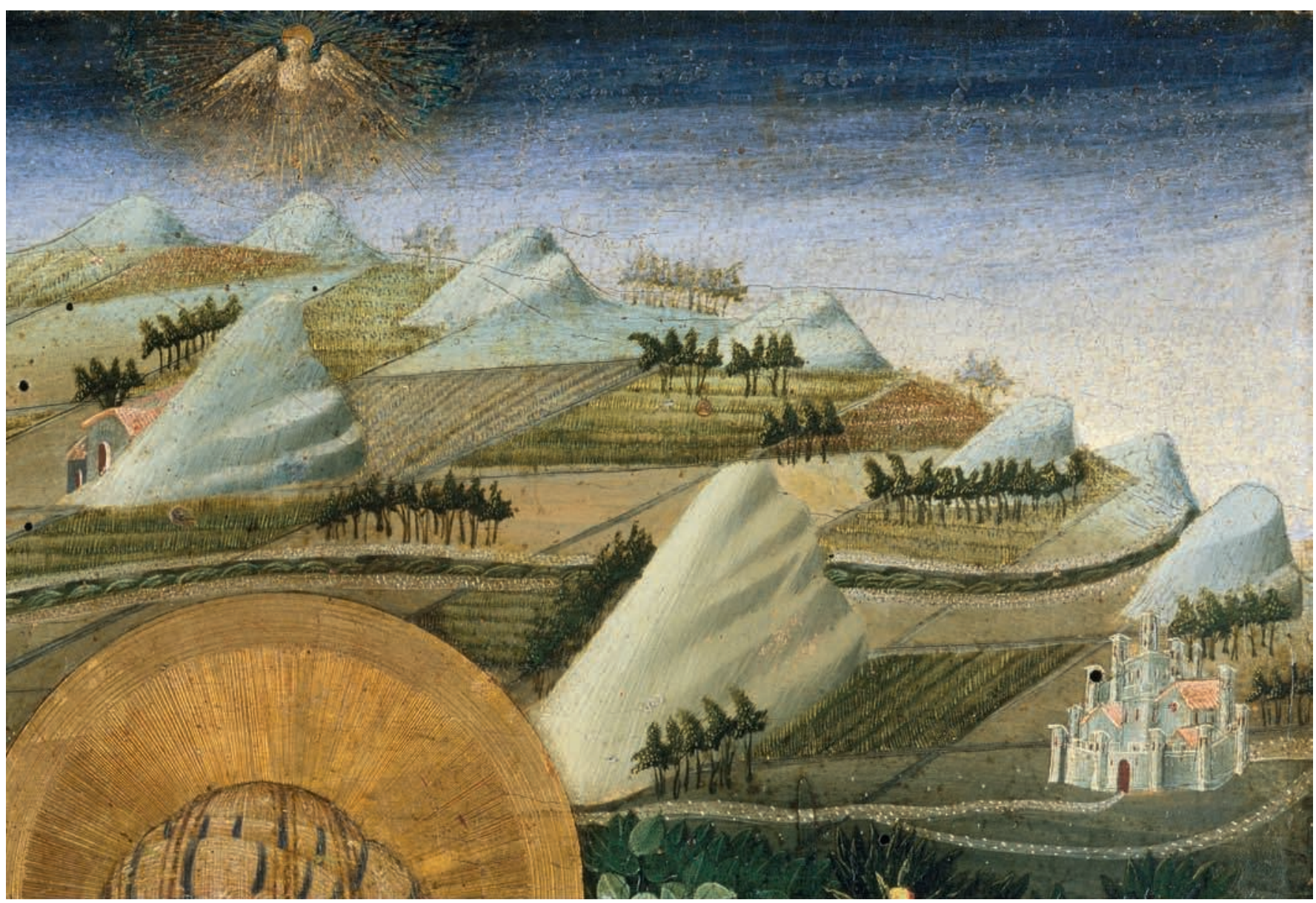

fig. 6

Giovanni di Paolo, Vierge à

l'enfant, 1440-1445 (détail),

Museum of Fine Arts, Boston. 
40. WHC-96/CONF.201/21, p. 66.

41. WHC-97/CONF.208/17, p. 47.

42. WHC-08/32.COM/24, p. 178.

43. SC-85/CONF.007/9, p. 11.

44. WHC-97/CONF.208/17, p. 42.

45. http://whc.unesco.org/ archive/advisory_body evaluation/345.pdf, p. 28.

46. WHC-96/CONF.201/21, Annex VI.2.

47. WHC-08/32.COM/ INF.8B1, p. 187.

48. WHC-96/CONF.201/21, Annex VI.2.

49. WHC-05/29.COM/ INF.22, p. 184-185. étant donné que ce n'étaient pas les édifices mais le paysage dans son ensemble qui était pris en compte. Mais dans le cas de certaines inscriptions récentes, des rénovations datant de l'époque romantique ont fait l'objet d'une réhabilitation complète: elles témoigneraient d'une étape importante dans l'histoire de la conservation du patrimoine et renforceraient la VUE du bien en question au lieu de l'amoindrir. C'est le cas des monuments commémoratifs de Luther à Wittenberg et à Eisleben en Allemagne ${ }^{40}$ (1996), du château de l'ordre teutonique de Marienbourg (Malbork) en Pologne 41 (1996) et du centre historique de Saint-Marin 42 (2008).

L'un des exemples les plus remarquables est le projet favori de Violletle-Duc à Carcassonne. L'architecte passa en effet plusieurs décennies à rénover et reconstruire la partie haute de l'enceinte de la ville, ainsi que la cathédrale. En 1985, une première proposition fut rejetée sur la base de l'inauthenticité du site ${ }^{\mathbf{4 3}}$. Mais dans une seconde proposition faite en 1997, on a jugé que les rénovations représentaient en réalité un moment décisif dans l'histoire de la conservation $\mathbf{4 4}^{4}$ et le site fut finalement inscrit sur la liste. Dans ce cas ${ }^{\mathbf{4 5}}$, comme dans ceux du château de Marienbourg ${ }^{\mathbf{4 6}}$ et de Saint-Marin ${ }^{47}$, les évaluations de l'Icomos et les conclusions des délégués se référaient explicitement au document de Nara. La délégation polonaise se félicita de la prise de distance avec «notre fondamentalisme ouesteuropéen [...] qui nous a amenés à la définition de la notion d'authenticité, limitée exclusivement à la substance matérielle; une idée que nous avons voulu octroyer aux autres régions culturelles du monde ${ }^{\mathbf{4 8}}$ ». La seule autre référence au document de Nara que j'ai pu trouver dans les documents du patrimoine mondial figure dans les notes de la longue discussion autour de la proposition d'inscription de Mostar ${ }^{49}$. Néanmoins, aucune ne mentionne la protection des structures de bois et de terre pour laquelle le document avait initialement été adopté. C'est là, me semble-t-il, une des conséquences imprévues liées à l'extension des critères d'authenticité.

\section{Biens en série}

Pour finir, la place de plus en plus grande des aspects immatériels au sein du patrimoine mondial se manifeste également à travers la popularité croissante des biens en série (Orientations, § 137-139). II s'agit de biens composés de deux ou plusieurs éléments - voire des centaines - séparés dans l'espace, dont l'importance ne tient pas à l'un d'eux mais à l'ensemble complet. Ils sont considérés comme un seul et même bien, et doivent donc être administrés comme tel à travers la mise en place d'un système de gestion coordonné, qui constitue une précondition à l'inscription (§ 114). Ils peuvent tout simplement consister en un ensemble de sites semblables, à l'instar des 11 forts et châteaux de la traite négrière situés au Ghana (voir plus haut), des 17 monuments historiques de l'ancien Kyoto (1994) ou encore, en Espagne, des quelque 727 sites d'art rupestre préhistorique (1998). Dans certains cas, les éléments isolés ne peuvent pas exister de manière indépendante. Le plus classique est celui de l'arc géodésique de Struve (2005) qui contribua à déterminer, au XIX siècle, la vraie forme elliptique de la Terre. Pris isolément, les 34 points fixes principaux (sur un total de 265 à l'origine) n'ont pas de sens; ils doivent être considérés dans leur ensemble. Répartis sur 12 pays, de la Norvège à la mer Noire, ils constituent jusqu'à présent le bien le plus étendu au monde. 
II faut évidemment avoir beaucoup d'imagination pour apprécier ce genre de «bien" car, lorsque les visiteurs se trouvent à l'un des points de l'arc, c'est moins le site en lui-même qu'ils considèrent que l'histoire liant entre eux les sites de la série. Cependant, malgré les critiques émises à l'encontre des biens en série et de certaines applications de ce principe de classement, de nouvelles candidatures continuent d'être présentées. En 2009, le Comité a examiné les premières propositions concernant des sites transcontinentaux: il s'agissait d'un ensemble de trois lieux, au Mexique, en Espagne et en Slovénie, historiquement reliés par le commerce du mercure (lui-même en lien avec la production d'argent), ainsi que d'un ensemble d'œuvres architecturales réalisées par Le Corbusier dans cinq pays européens et au Japon. Ces propositions firent immédiatement apparaître des difficultés. En effet, l'un des éléments de la route du mercure - San Luís Potosí au Mexique - faisait également partie d'un autre projet de bien en série que le Mexique souhaitait présenter à la session de 2010. Les délégués s'interrogèrent donc sur la façon dont ils devaient évaluer un site relevant de deux projets distincts. Par ailleurs, des biens en série avaient déjà réuni les œuvres d'un même architecte: elles étaient généralement rassemblées en un même lieu - les œuvres d'Antoni Gaudi à Barcelone (1984 et 2005) - ou bien remplissaient la même fonction - les fortifications de Vauban (2008) -, ou encore les deux à la fois - les habitations majeures de Victor Horta à Bruxelles (2000). Mais dans le cas des œuvres du Corbusier, qui constituaient un ensemble extrêmement diversifié rassemblant des réalisations de second ordre tout en laissant de côté des ouvrages majeurs (Chandigarh en Inde, par exemple), elles n'étaient liées que par le nom de leur créateur. Les opposants à ce type de bien arguèrent que cela risquait de donner lieu à des propositions similaires, telles les œuvres de Frank Lloyd Wright qui figuraient déjà en tant que futures candidates sur la «liste indicative» des États-Unis. Après de vifs débats, les propositions concernant la route du mercure et Le Corbusier furent toutes deux renvoyées aux États à l'initiative de la proposition. Lors d'une nouvelle présentation en 2010 et 2011, elles furent encore rejetées, l'Icomos n'étant toujours pas satisfait de la manière dont le concept de série était utilisé. Le projet autour de la route du mercure a finalement été inscrit sur la liste en 2012 sous le nom de "Patrimoine du mercure: Almadén et Idrija", sans l'élément mexicain, ce qui rend la série plus cohérente et moins étirée géographiquement. La proposition Le Corbusier est quant à elle toujours en attente. En outre, deux projets de séries gigantesques, comprenant des dizaines d'éléments et s'étendant sur plusieurs pays, sont en cours de réalisation: la route de la soie en Asie et le Qhapaq Ñan, la grande route inca qui traverse les Andes. Bien que, dans tous les cas exposés ici, les éléments pris individuellement soient tout à fait matériels et même, pour certains d'entre eux, relativement célèbres, ils ne représenteraient pourtant pas grand-chose en dehors de la série où ils sont rassemblés. Comme dans les autres exemples évoqués, la beauté des conceptions l'emporte sur celle de chaque site.

\section{Les causes du passage à l'immatériel}

Comment expliquer que les aspects immatériels prennent de plus en plus d'importance dans les discours et les pratiques des acteurs du patrimoine mondial? Cela est en partie dû à un changement d'attitude plus général. Les responsables des questions de patrimoine s'accordent sur 
fig. 7

L'arc géodésique de Struve, Baelljasvarri. Kautokeino, Norvège. L'arc de Struve est un réseau de triangulations qui a contribué à définir et mesurer la taille et la forme exactes de la Terre et qui s'étend de Hammerfest en Norvège jusqu'à la mer Noire et traverse 10 pays. Le site inscrit en 2005 comprend 34 des points fixes d'origine. (C) Unesco.
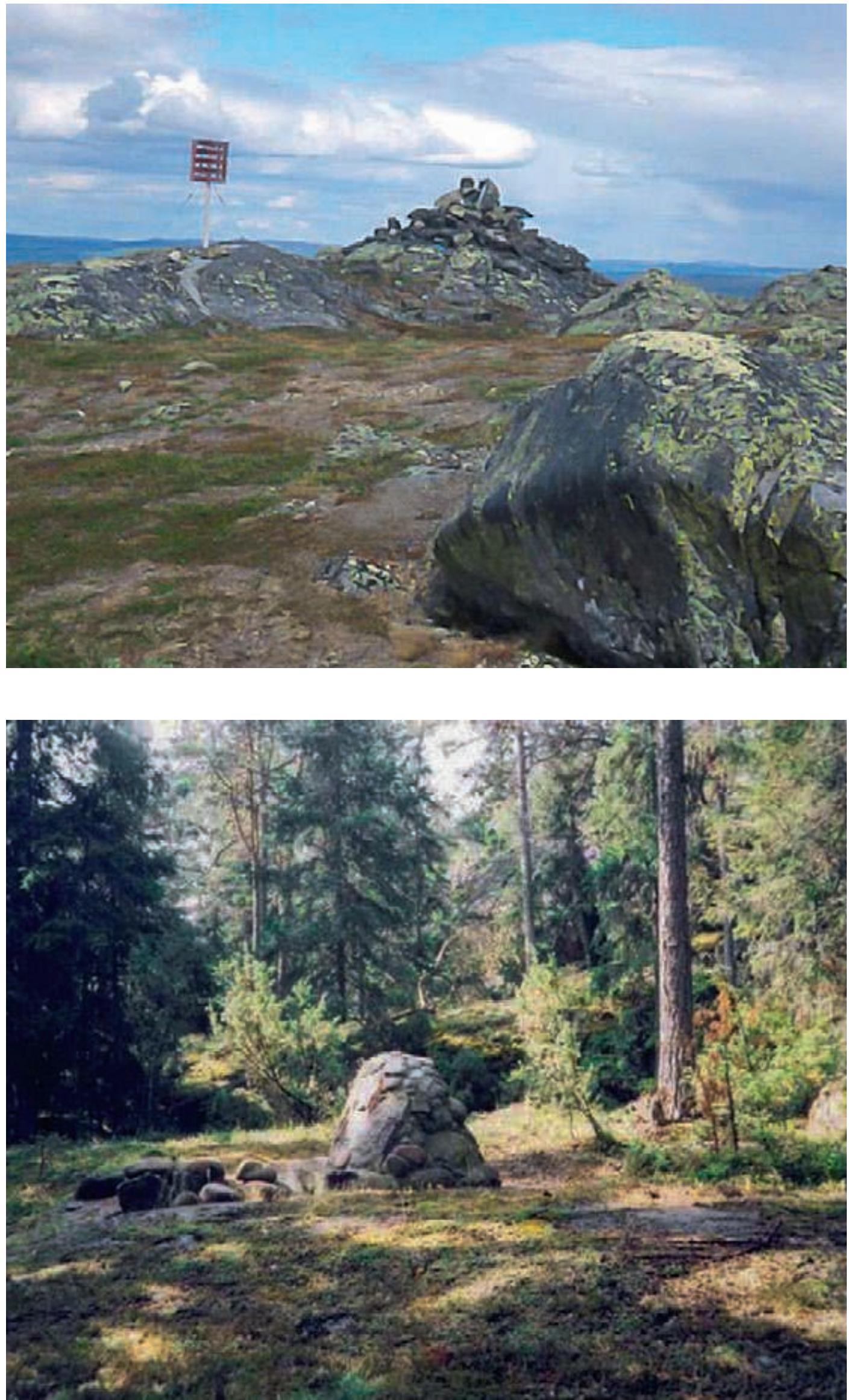

L'arc de Struve, Gogland, Russie. () Unesco. 

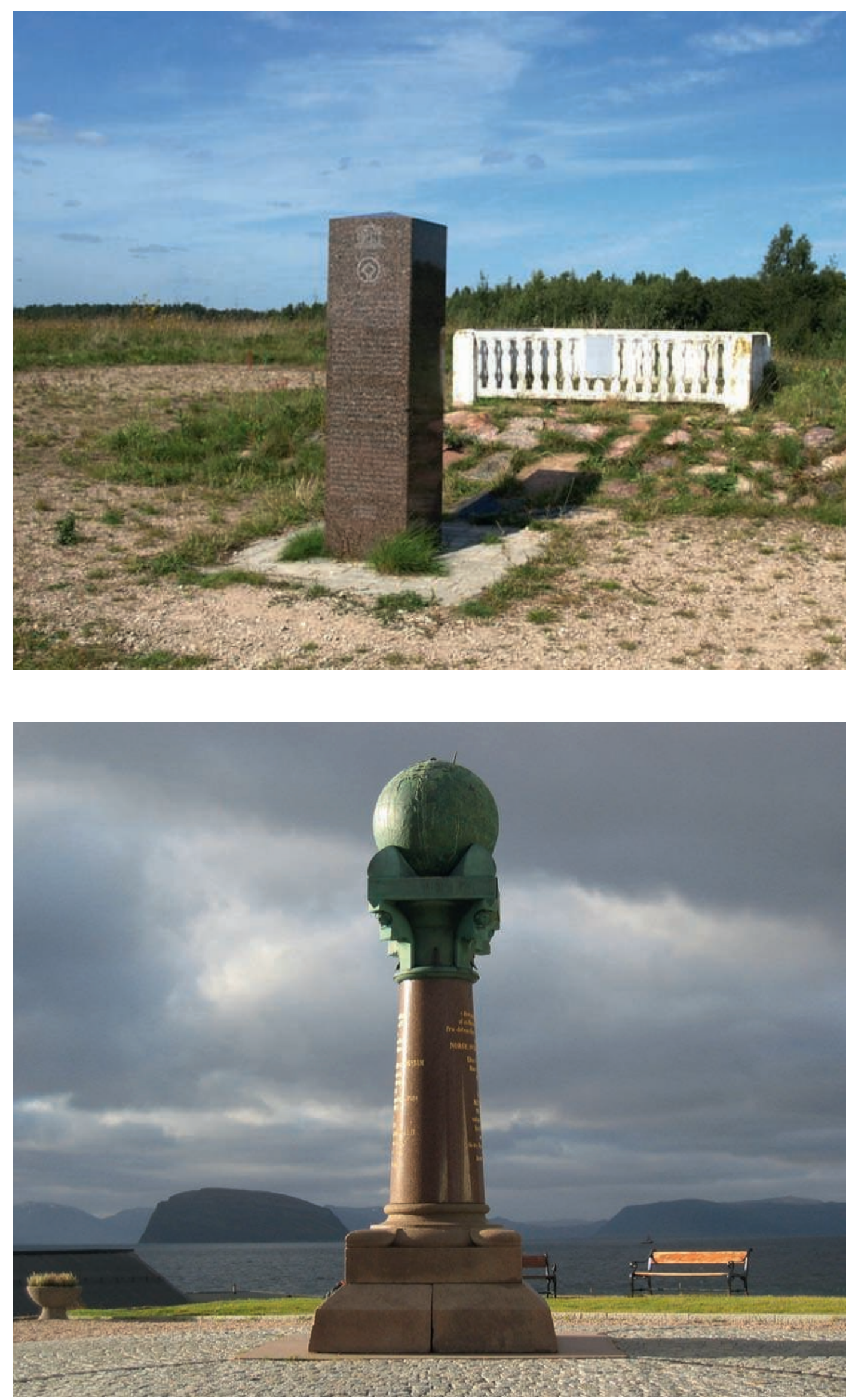

fig: 9

L'arc de Struve à Beresnäki, Nemezis, Lithuanie. (C) Unesco. fig. 10

Le monument de l'arc du méridien, Hammerfest,

Norvège. (๑) Francesco Bandarin/ Unesco. 
le fait que la dimension immatérielle a jusqu'à présent été négligée, et ce consensus a peu à peu fait son chemin jusqu'aux sphères décisionnelles du patrimoine mondial. Par ailleurs, les États les mieux représentés ont fait le tour de leurs candidats les plus attendus, ce que confirmerait une simple comparaison entre les premières inscriptions au patrimoine mondial sollicitées par les États d'Europe de l'Ouest et les candidatures figurant aujourd'hui sur leurs «listes indicatives». Les arguments en faveur de ces sites doivent dès lors se fonder sur autre chose que leur aspect physique, qui est souvent assez peu remarquable.

Les nouvelles orientations représentent également, à bien des égards, une réaction à la Convention de 2003 sur le patrimoine culturel immatériel ainsi qu'au programme plus ancien des «Chefs-d'œuvre du patrimoine oral et immatériel». Bien que ce soit le programme du patrimoine mondial qui continue de susciter le plus la convoitise, la Convention de 2003 séduit par sa nouveauté et reçoit les faveurs des médias, comme l'a montré, en 2010, le soutien public du président Sarkozy au projet d'inscrire le «repas gastronomique des Français» sur la liste représentative. Par comparaison, le patrimoine mondial apparaît quelque peu démodé, et certains experts proches de celui-ci expriment des réserves vis-à-vis du patrimoine culturel immatériel. J'ai pu le constater auprès de mes informateurs et Britta Rudolff l'a observé à l'occasion de plusieurs réunions très tendues destinées à établir des passerelles entre les deux camps (Rudolff 2010: 41-43). Dans une certaine mesure, cette rivalité reflète des frontières disciplinaires: architectes, historiens de l'art et archéologues dominent le discours d'expertise et les organisations consultatives du côté du patrimoine mondial (culturel), tandis que l'on tend davantage à consulter des anthropologues, des spécialistes du folklore et des musicologues pour le patrimoine culturel immatériel. Cela pourrait en théorie provoquer une rupture plus nette qui aboutirait à l'exclusion complète de toute dimension intangible du patrimoine mondial. Cependant, aux yeux des diplomates non spécialistes à la tête des délégations étatiques lors des réunions des conventions comme pour bon nombre de responsables politiques de chacun des pays impliqués ainsi que pour le grand public, qui continue de confondre les processus en utilisant indistinctement le terme de patrimoine mondial pour parler de listes différentes, les domaines couverts par les deux textes sont liés entre eux. Ainsi, ce qui est célébré d'un côté ne peut tout bonnement pas être disqualifié de l'autre: il est donc peu probable que les aspects immatériels perdent de leur importance au sein du système du patrimoine mondial. L'Icomos lui-même a consacré son assemblée générale de 2009 à la question de "L'esprit du lieu ", préférant le compromis à l'opposition frontale.

Cependant, plus encore que le souci de cohérence intellectuelle, c'est la tension permanente entre les aspirations universalistes du patrimoine mondial, d'un côté, et les intérêts particuliers des États-nations, de l'autre, qui joue un rôle fondamental. Comme toutes les autres institutions multilatérales, l'Unesco conçoit le monde comme la somme de ses parties, à savoir les États-nations membres de l'Organisation. Seuls ces derniers ont le droit de ratifier la Convention du patrimoine mondial et de proposer des sites pour inscription. Le texte a certes donné pouvoir à des organisations consultatives, l'Icomos, l'Union internationale pour la conservation de 
la nature (UICN), qui est consultée pour les sites naturels, et le Centre international d'études pour la conservation et la restauration des biens culturels (Iccrom), qui organise à Rome des formations et des stages de renforcement des compétences, chargées d'évaluer toutes les candidatures, en grande partie sur le modèle de l'évaluation par les pairs dans l'univers académique. Cependant, toutes les décisions sont, en dernière instance, prises par le Comité du patrimoine mondial, qui réunit les délégués de 21 États élus lors de l'Assemblée générale biannuelle de tous les «États parties " (c'est-à-dire ayant ratifié la Convention). Les États du Comité et les États non membres qui assistent souvent aux sessions en tant qu'observateurs sont absolument souverains pour décider de leur stratégie interne: ce sont eux qui choisissent quels délégués (en général des équipes très nombreuses) envoyer aux sessions du Comité, s'il faut laisser la parole à un expert du patrimoine ou, comme cela se fait de plus en plus, à un diplomate, quels sites proposer, dans quel ordre, lesquels laisser de côté, etc. Dans les sessions, la parole est toujours donnée à un État, jamais à un individu en particulier, et les délégations reçoivent des ordres de leur ministère pour intervenir au moins sur certains points à l'ordre du jour, ce qui limite leur engagement universaliste. Pour la plupart des États parties, l'objectif fondamental est d'abord de faire aboutir leurs candidatures nationales et, dans un second temps, de protéger ces projets d'une quelconque ingérence de la part du Comité une fois l'inscription réalisée. La meilleure manière de procéder est bien souvent de pratiquer un lobbying intense et de passer des accords avec les États siégeant au Comité en échange d'un soutien lors des autres assemblées de l'Unesco, des Nations unies, ou bien au cours de négociations bilatérales. D'après le témoignage de mes informateurs, ces transactions sont monnaie courante et expliquent un certain nombre d'interventions qui n'auraient sinon aucun sens. Les conflits d'intérêts sont particulièrement forts quand les États du Comité eux-mêmes présentent des candidatures, une pratique qui, n'étant pas interdite, leur permet de faire aboutir un nombre disproportionné d'inscriptions. Les appels à la retenue de la part des États membres n'ont jamais eu d'effets à long terme, et toutes les tentatives pour mettre en place des moratoires ou des quotas de nominations n'ont eu qu'une portée limitée.

À ce constat s'ajoute le fait que, au-delà des efforts très maigres déjà évoqués, telle la décision de qualifier Auschwitz de cas exemplaire, le Comité n'est pas coutumier des décisions préventives; il a plutôt tendance à traiter les problèmes généraux posés par des cas particuliers. En théorie, il serait par exemple envisageable d'exclure des types spécifiques de sites tels que les champs de bataille avant même qu'ils ne soient proposés à l'inscription. Dans la pratique, rien de cela ne se produit, les États pouvant dès lors faire preuve d'une très grande inventivité dans leurs propositions. II n'existe pas non plus de tradition consistant à prendre en compte, dans l'évaluation, la performance de l'État partie - par exemple le nombre de biens inscrits au patrimoine mondial ou encore le résultat de sa politique en matière de conservation. En outre, la VUE fait figure de critère absolu, bien que les efforts de définition opérationnelle ${ }^{\mathbf{5 0}}$ de la notion puissent sembler quelque peu insuffisants pour un œil critique. Si le nombre de nouvelles propositions est certes limité, aucune barre n'est fixée pour les inscriptions annuelles ni pour le total d'entre elles. Théoriquement, les 1497 candidatures
50. WHC-08/32.COM/9. 


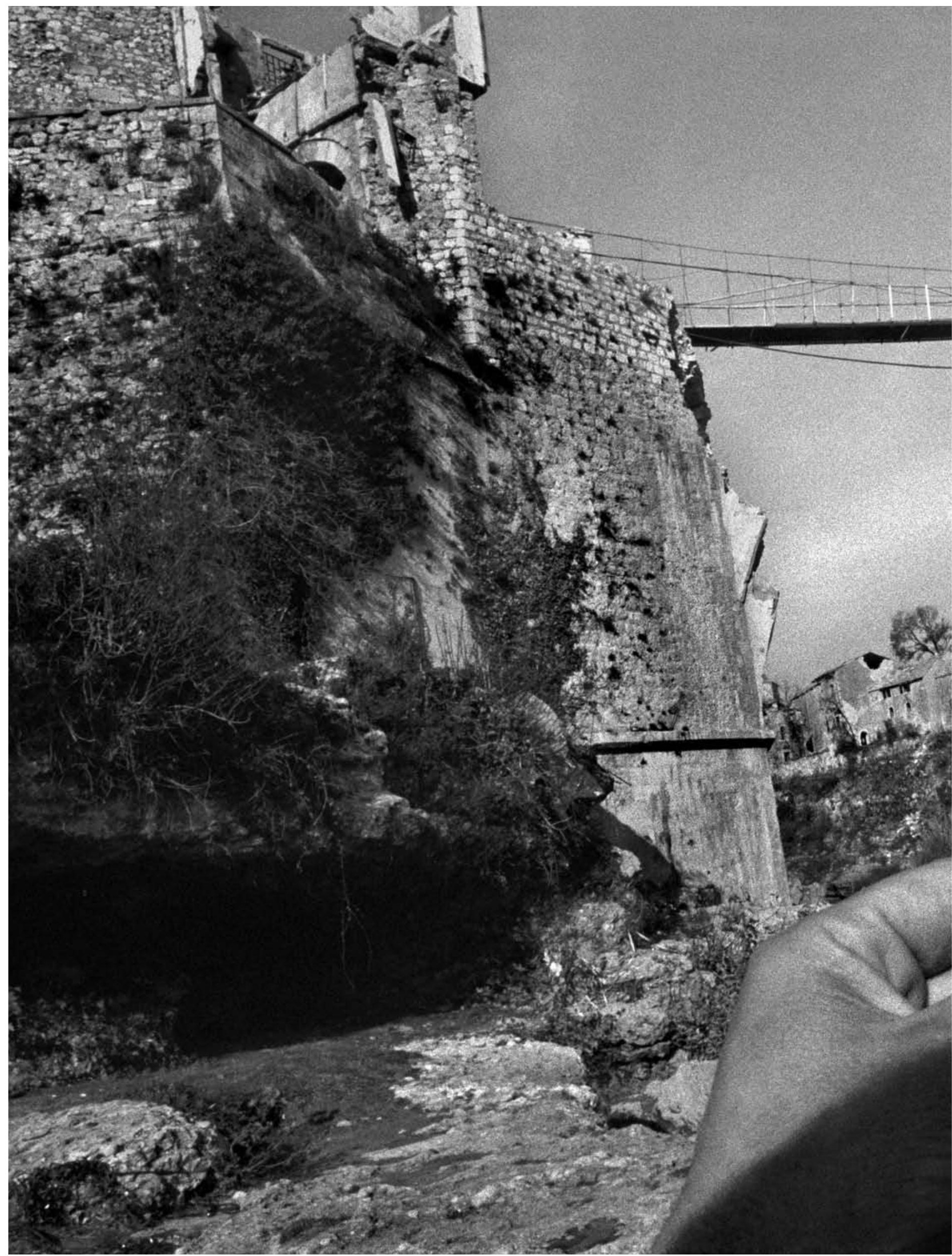


51. WHC-94/CONF.003/ INF.6.

52. 25 C/33; 25 C/Reports, p. 36.

53. Un exemple nous est fourni par le discours de Koïchiro Matsuura, directeur général de l'Unesco, prononcé lors de la première Assemblée générale de la nouvelle Convention:

"À l'époque où je présidais le Comité du patrimoine mondial [1998-1999], j'étais très conscient de la répartition géographique inégale des sites de la liste du patrimoine mondial, qui était, de manière générale, plus représentative du "Nord". Ce déséquilibre de fait reflétait une faiblesse dans notre système: uniquement préoccupé par la protection du patrimoine matériel, il ignorait le patrimoine immatériel et laissait ainsi de côté des aspects culturels qui jouent pourtant un rôle fondamental dans la diversité culturelle bien souvent incarnée par les cultures du "Sud"." (DG/2001/52, p. 1)

\section{double page précédente}

fig $=11$

Josef Koudelka, Le pont de Mostar, 1994. (C) JK/Magnum Photos. potentielles qui se bousculent sur les listes indicatives des États parties pourraient toutes figurer un jour sur la liste du patrimoine mondial. Et s'il n'y a pas de raison de douter de leur VUE, c'est ce qui se passera en effet comme ne se lassent pas de le souligner les États, toujours avides de nouvelles inscriptions.

Dans de telles conditions, la liste fait aujourd'hui la part belle aux pays d'Europe occidentale et d'Amérique du Nord, qui bénéficient de budgets et de contingents de spécialistes beaucoup plus importants que les autres, ainsi qu'à des États tout aussi intéressés et dynamiques tels que les pays d'Asie de l'Est et le Mexique. Cependant, il devient extrêmement difficile de justifier, pour un projet à visée universaliste comme celui de l'Unesco, le fait que l'Europe héberge au moins dix fois plus de biens par habitant que l'Asie et que, en outre, l'Afrique subsaharienne possède si peu de sites culturels. Face à cette réalité, nombreux sont ceux qui ont dénoncé la faible "représentativité" de la liste, en particulier à l'égard des pays du Sud, ce qui a conduit à l'adoption en 1994 d'un projet de réforme de la Stratégie globale ${ }^{51}$. Ce document programmatique rejette la vision occidentale conventionnelle du patrimoine et la survalorisation des palais, des églises et des centres-villes historiques, et propose plusieurs domaines thématiques nouveaux "dans leur contexte anthropologique général». Les critiques à l'encontre de l'eurocentrisme de la liste du patrimoine mondial sont aussi très largement à l'origine du programme des "Chefsd'œuvre", devenu ensuite la Convention sur le patrimoine culturel immatériel. Si d'autres facteurs ont joué un rôle (Schmitt 2008; Bortolotto 2010), ces critiques ont, selon moi, été les plus déterminantes pour décider I'Unesco à aller plus loin que ne le faisait un texte antérieur et moins contraignant, les « Recommandations sur la sauvegarde de la culture traditionnelle et populaire", adopté en $1989^{52}$. De nombreux défenseurs de la nouvelle Convention souscrivent à une logique compensatoire, qui va souvent de pair avec certaines conceptions simplistes de la distribution géographique des différentes formes de patrimoine ${ }^{53}$.

Toutes ces évolutions ont sans nul doute contribué à accueillir des sites d'un type nouveau sur la liste du patrimoine mondial. Moins élitiste, celle-ci est désormais plus "anthropologique» et prend en compte les traces matérielles de la vie quotidienne. Cependant, presque la moitié du total des inscriptions concerne encore l'Europe; de plus, les pays très actifs en termes de propositions sont également les plus rapides quand il s'agit de s'adapter aux nouvelles catégories. L'Italie, par exemple, est première de la liste non seulement en nombre de sites répertoriés (47), mais également de paysages culturels (5). La percée réalisée par l'«immatériel» au sein du patrimoine mondial est dès lors autant le produit de changements programmatiques que de l'adaptabilité d'États anxieux de figurer sur la liste (et avec eux certaines régions et villes), qui saisissent toutes les occasions de concevoir de nouvelles candidatures adaptées à un label dont on attend des miracles pour l'industrie touristique et la valorisation du pays. Et si l'heure est à la réhabilitation de l'intangible, la résistance aux propositions élaborées pour correspondre à cette évolution est faible; le tournant politique et le changement de mentalité qui semblent en résulter sont moins réels qu'il n'y paraît. 
Cela est particulièrement évident dans le cas des biens en série évoqués plus haut. Les Orientations établissent que, tant que la série dans son ensemble possède une VUE, les éléments isolés qui la composent n'ont pas nécessairement besoin d'en avoir une (§ 137); un tel raisonnement est particulièrement adapté aux biens tels que l'arc géodésique de Struve cité plus haut. Mais cette condition est parfois utilisée pour rattacher à une série des éléments qui n'auraient autrement pas pu être facilement défendus devant les organisations consultatives et le Comité. C'est précisément ce que l'Icomos a reproché à certains éléments du projet mexicain du Camino Real de Tierra Adentro (inscrit en 2010 malgré l'avis défavorable de l'Organisation ${ }^{54}$ ). Cette logique a également été invoquée par mes informateurs pour expliquer les «ajouts" auxquels procèdent les États: ces derniers peuvent ainsi augmenter une série - par exemple en additionnant 24 beffrois français et belges (2005) à la liste des 33 déjà existants en Belgique -; ils peuvent transformer des sites isolés en biens en série - comme ce fut le cas lorsque le chemin de fer de montagne des Nîlgîri (2005) et la ligne de Kalka à Shimla (2008) vinrent rejoindre la liste déjà établie du chemin de fer himalayen de Darjeeling (1999), inaugurant ainsi la série des «Chemins de fer de montagne en Inde", ou quand le limes de Germanie (2005) et le mur d'Antonin en Angleterre (2008) furent ajoutés au mur d'Hadrien (1987), créant ainsi les "Frontières de l'Empire romain", ou encore avec le projet actuel de réunir dans un même bien transnational un certain nombre de sites vikings figurant déjà sur la liste à titre individuel et de nouveaux sites. Ces séries transfrontalières sont très prisées puisque, au titre de l'amitié internationale qu'elles sont censées exprimer, elles ne sont pas comptabilisées dans les quotas de propositions, ce qui permet aux acteurs les plus dynamiques de dépasser la limite habituellement autorisée (qui est, à l'heure actuelle, d'un site et d'un paysage culturel par an). Bien que les partenariats noués en toute bonne foi autour de ces projets de séries puissent favoriser la connaissance et la conservation des sites en question, l'impulsion de départ vient souvent de ce rouage de la procédure que les États européens exploitent très sciemment, eux qui bénéficient d'une longue expérience de la coopération avec leurs nombreux voisins.

La Convention de 2003 a suivi une évolution tout à fait semblable, ce qui va dans le sens de mon hypothèse selon laquelle la motivation principale est avant tout de figurer sur la liste. Contre l'avis de presque tous les experts consultés, elle a repris la même structure générale que celle du patrimoine mondial: seuls les délégués des États parties prennent les décisions (à la différence du programme des "Chefs-d'œuvre" pour lequel un jury d'experts procédait à la sélection), les États membres du Comité ont le droit de proposer leurs propres sites à l'inscription durant leur mandat et le Comité gère une «liste représentative", cette dernière faisant manifestement l'objet de toutes les convoitises en dépit des recommandations qui suggèrent de privilégier la «liste du patrimoine culturel immatériel nécessitant une sauvegarde urgente ». La Convention de 2003 a simplement laissé de côté les freins éventuels aux appétits nationaux: elle ne prévoit pas d'organisations consultatives suffisamment fortes pour contrecarrer les propositions d'inscription, et n'a pas non plus fixé de palier qualitatif à l'instar de la VUE. Étant donné leur longue expérience en matière de conservation du patrimoine immatériel, les États d'Asie de l'Est sont
54. WHC-10/34.COM/ INF.8B1.Add, p. 107-114. 
55. WHC-11/18.GA/INF.8 particulièrement bien préparés et ont inscrit des sites par dizaines, dépassant ainsi les pays européens; les pays africains, à l'attention desquels la Convention avait avant tout été rédigée, sont encore une fois loin derrière. Et là aussi, les débats autour de la représentativité et des quotas ont été au cœur des dernières sessions du Comité.

Entre 2010 et 2013, certains pays puissants du Sud ont commencé à prendre les choses en main lors des sessions du Comité du patrimoine mondial, conséquence logique de la frustration accumulée au cours des dernières années. De façon inédite, ces pays membres ont délibérément ignoré les recommandations des organisations consultatives, si bien que presque toutes les candidatures qui avaient fait l'objet d'un travail préalable de lobbying ont été validées. De la même manière, les inscriptions sur la «liste du patrimoine mondial en péril» proposées par les organisations consultatives et le secrétariat ont été rejetées sans autre forme de considération à chaque fois qu'elles allaient à l'encontre de la volonté d'un de ces pays, et ce pour éviter toute situation embarrassante. Ces pratiques ont été vivement critiquées par la presse (The Economist, 26 août 2010) et par les États non membres du Comité. Par la suite, et en réponse à un audit externe très négatif de la Stratégie globale ${ }^{55}$, l'Assemblée générale de 2011 a mis en place un groupe de travail chargé de proposer des éléments de réforme. En dépit de toutes les controverses soulevées, les sessions de 2010 et 2011 ont répondu au moins à l'un des objectifs de la Stratégie globale: les inscriptions européennes et nord-américaines ont atteint proportionnellement et respectivement leur deuxième et quatrième chiffre le plus bas dans I'histoire de la Convention depuis 1978.

\section{Conclusion}

L'avenir nous dira l'impact de cette nouvelle évolution sur l'importance de plus en plus grande prise par l'immatériel. Cependant, il apparaît clairement que les aspects intangibles des sites matériels ont gagné du terrain au sein du système du patrimoine mondial. Le critère (vI), qui met à l'honneur les liens avec des idées, des événements ou des personnages importants, a peu à peu été renforcé; depuis les années 2000, en particulier, il a été utilisé pour définir la VUE de sites «sombres » de commémoration (et parfois aussi de réconciliation) très chargés sur le plan politique: un camp de concentration, des cibles nucléaires, des sites détruits de manière délibérée, etc. Les controverses autour des associations avec des personnages célèbres ou des croyances religieuses, qui tendent à éclipser les aspects matériels des sites en question, ont donné lieu à des conclusions différentes et pas toujours cohérentes; le débat n'est manifestement pas encore terminé. La réhabilitation des dimensions immatérielles, telles les traditions orales dans le cas des paysages culturels, a, de manière générale, déclenché moins de controverses, notamment pour la sous-catégorie des paysages culturels associatifs où les traces matérielles des associations en question ne sont pas requises. De manière moins évidente, l'accent mis sur les idées plutôt que sur la matérialité des sites est également caractéristique de la tolérance de plus en plus grande à l'égard des restaurations et des rénovations que l'on jugeait autrefois inauthentiques et que l'on considère de nouveau aujourd'hui comme une étape importante dans I'histoire de la conservation. Enfin, le passage à l'immatériel est également visible dans la popularité 
croissante des biens en série, qui sont parfois transnationaux et dont la VUE réside dans les événements, les idées et les relations fonctionnelles qui lient les sites entre eux plutôt que dans la valeur indépendante de chacun et de son aspect extérieur.

Cette évolution reflète une tendance plus large à la reconnaissance de la dimension immatérielle du patrimoine. Celle-ci est renforcée par la Convention de 2003 pour le patrimoine culturel immatériel et par la pression ainsi créée en faveur de la légitimation de ces biens, et par la difficulté toute simple à envisager, d'un point de vue conceptuel, les deux conventions de I'Unesco comme des entités distinctes, en particulier pour les non-spécialistes comme les diplomates de carrière qui se trouvent bien souvent à la tête des délégations étatiques dans les deux comités. Cependant, c'est avant tout l'avidité de nombreux États, régions et municipalités qui explique fondamentalement cette tendance, en particulier depuis qu'on a montré combien il était utile sur un plan politique et économique de figurer sur la liste du patrimoine mondial. C'est là, selon moi, ce qui explique l'exploitation de nouvelles possibilités quand celles-ci semblent jouer en faveur de candidatures qui n'avaient auparavant aucun espoir d'être retenues. Dès lors, ce qui ressemble de loin à un changement de politique conscient n'est en réalité qu'une rationalisation a posteriori des conséquences de l'opportunisme de certains États. À partir du moment où l'immatériel semble fournir des voies d'accès variées au patrimoine mondial, il est rapidement exploité, bien souvent par des acteurs très sceptiques sur l'intérêt du concept.

Max Planck Institute for Social Anthropology, Halle brumann@eth.mpg.de 
Bibliographie

\section{Bortolotto, Chiara}

2010 " Globalising intangible cultural heritage? Between international arenas and local appropriations ", in Sophia Labadi et Colin Long (éd.),

Heritage and Globalisation. Londres, Routledge: 97-114.

\section{Fowler, Peter J.}

2003 World Heritage Cultural Landscapes, 1992-2002. Paris, Unesco World Heritage Centre.

\section{Logan, William Stewart et Reeves, Keir (éd.)}

2008 Places of Pain and Shame Dealing with "difficult heritage". Londres, Routledge.

\section{Rudolff, Britta}

2010 "Intangible" and "Tangible" Heritage. A Topology of Culture in Contexts of Faith.

Bonn, Scientia Bonnensis.

\section{Ruskin, John}

1987 [1849] Les Sept Lampes de l'architecture. Paris, Denoël.

\section{Schmitt, Thomas M.}

2008 "The Unesco concept of safeguarding intangible cultura heritage: Its background and Marrakchi roots ", International Journal of Heritage Studies 14 : 95-111.

2009 " Global cultural governance: Decision making concerning World Heritage between politics and science", Erdkunde 63: 103-121.

2011 Cultural Governance: Zur Kulturgeographie des Unesco-Welterberegimes. Stuttgart, Franz Steiner Verlag.

\section{The Economist}

2010 "A danger list in danger" 26 août; http://www.economist. com/node/16891951.

\section{Comment le patrimoine mondial de I'Unesco} devient immatériel. Par Christoph Brumann

\section{Titchen, Sarah M.}

1995 On the construction

of outstanding universal value.

Unesco's World Heritage

Convention (Convention concerning the Protection of the World Cultural and Natural Heritage, 1972) and

the identification and assessment of cultural places for inclusion in the World Heritage List.

Australian National University, thèse de doctorat.

\section{Turtinen, Jan}

2000 Globalising Heritage:

On UNESCO and the Transnational Construction of a World Heritage. Stockholm, Stockholms Universitet ("SCORE Rapportserie 12"); http://www.score.su.se (consulté le 6 août 2013)

\section{Väldsarvets villkor:} Intressen, förhandlinger och bruk $i$ internationell politik. Stockholm Universitet, thèse de doctorat.

\section{Viollet-le-Duc, Eugène}

1875 Dictionnaire raisonné de l'architecture française $d u \times l^{\ominus}$ au $\times v^{e}$ siècle, vol. VIII.

Paris, A. Morel.

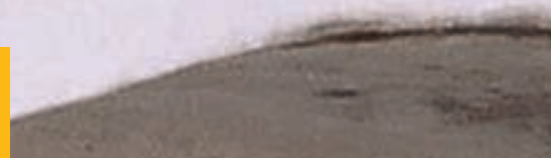

ci-contre

Le fort de Cape Coast. Spectacle donné à l'occasion du lancement de l'année de commémoration de la lutte contre l'esclavage et de son abolition, 2004, Ghana. (c) Bernard Jacquot/ Unesco.

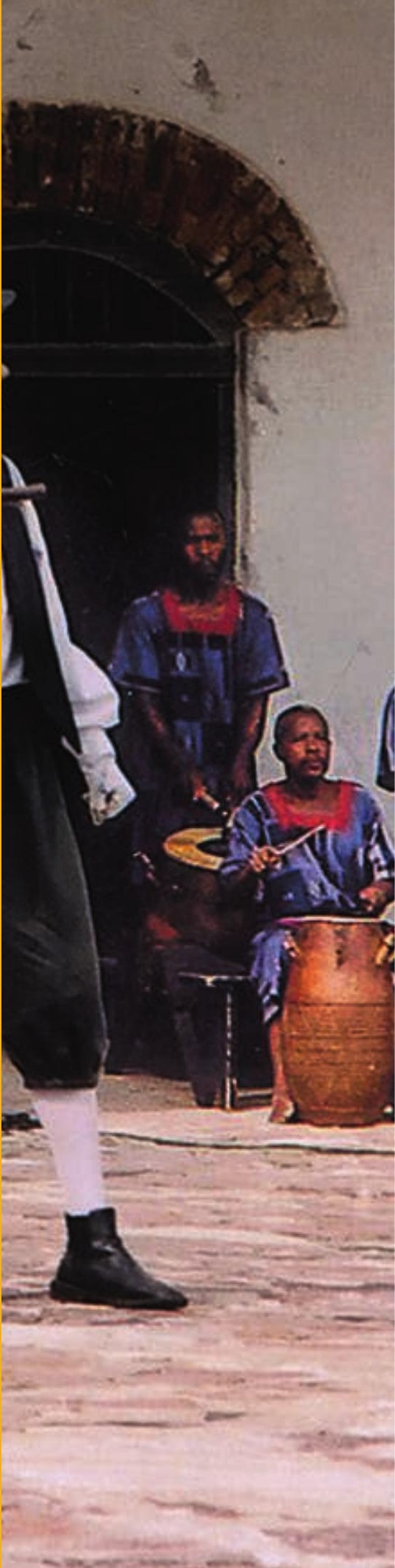




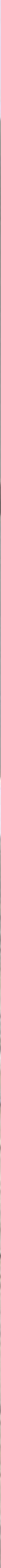

